\title{
Análisis estructural de la investigación empírica sobre el periodista latinoamericano ${ }^{1}$
}

\author{
Claudia MELlado 2
}

Este artículo reporta resultados preeliminares de un análisis secundario documental de la investigación empírica realizada sobre el periodista latinoamericano en el periodo 1960 2007. Se describe la evolución temporal de los estudios, su procedencia, el tipo de formato utilizado, las entidades difusoras más importantes, la tipología temática de las investigaciones, los niveles de análisis predominantes y los enfoques metodológicos utilizados. Los resultados enseñan la irregularidad de este tipo de estudios en Latinoamérica.

PALABRAS CLAVE: estudios sobre periodistas, análisis secundario, producción académica, Latinoamérica.
This article reports preliminary findings of a quantitative secondary analysis of Latin American journalists studies in the period 1960-2007. The temporary evolution of the studies, the country of publication, the type of publication, the most important publishers, the thematic typology of each empirical work, the levels of analyses addressed, and the methodological approaches were described. The results show the irregularity of this type of studies in Latin America.

KEY WORDS: journalist studies, journalism, secondary analysis, scholarly production, Latin America.

1 Este artículo es parte del trabajo del Proyecto de Investigación FONDECyT No. 1080066 (Chile).

2 Facultad de Ciencias Sociales de la Universidad de Concepción (Chile) Correo Electrónico: claudiamellado@udec.cl 


\section{INTRODUCCIÓN}

La producción académica es un elemento fundamental en la comprensión del desarrollo del conocimiento, y es hoy uno de los indicadores más claros respecto a lo que constituye el capital científico -como lo definiría Bourdieu-y los marcos de acción de un campo de estudio.

Para Meditsch y Segala (2005), una forma de unir el rigor científico con resultados relevantes dentro de un área de estudios es adquirir el hábito de repasar nuestras bibliografías de un modo más sistemático y riguroso, ser capaces de reconocer nuestros avances, así como de criticar nuestros métodos o resultados.

No obstante, en la búsqueda por describir el sistema social en el cual se produce el conocimiento científico en torno a la profesión periodística, y específicamente sobre los periodistas latinoamericanos, lo cierto es que poca atención se ha puesto a cómo dicho conocimiento crece y madura.

Parafraseando a Fuentes (1991, p.18), sea porque la investigación, la teoría, la profesión y la enseñanza de la comunicación son consideradas tácitamente como estructuras transparentes, o tal vez, al contrario, por la dificultad de desentrañarlas, la autorreflexión crítica y sistemática en este ámbito específico de estudio no parece aún haber aflorado lo suficiente.

Los tópicos de investigación han estado más orientados hacia el receptor, el medio o el mensaje por sí mismo, y no, en cambio, hacia el emisor como sujeto influenciado y condicionado por una realidad contextual, laboral y profesional que podría mediar en su capacidad de producir y generar contenidos y mensajes en el entorno profesional en que se ve inserto.

Menanteau-Horta (1967), precursor de estos estudios en el subcontinente, reconoció en su tiempo que:

Si una carencia de los estudios que conciernen al comunicador profesional es sentida en los países que sostienen una fuerte tradición en la investigación, la ausencia de información sobre el periodista es más crítica en países donde el interés sociológico sólo comienza (p. 716). 
Bajo la misma idea, Wright (1975, p. 60-61) enfatiza que el estudio sobre los comunicadores, a nivel mundial, ha sido descuidado para dar atención investigativa a los demás componentes del proceso de comunicación. Asimismo, recuerda que la mayor parte del conocimiento existente sobre los periodistas y comunicadores proviene de documentos descriptivos como novelas, biografías, historias e informes periodísticos sobre los medios, por lo que es necesario dar la bienvenida a una narrativa adicional, a partir de análisis sociográficos y sociológicos, que revelen cómo los roles y actitudes del periodista y comunicador afectan el contenido que producen (ibidem, p. 76-77).

No es sino después la consolidación de los estudios vinculados al newsmaking (Tuchman, 1978), cuando el interés sobre este tipo de estudios comienza a aumentar de forma sustantiva. No obstante, estos suelen encontrarse dispersos y en un estado de desconocimiento notorio, al no haberse desarrollado aún una sistematización documental de la producción científica en torno a la figura del periodista latinoamericano y su profesión.

Una vez constatada dicha realidad, este reporte preeliminar de investigación nace desde la convicción de que existe una brecha entre la producción científica que se realiza sobre el periodista latinoamericano y el conocimiento que la propia academia tiene sobre ella.

\section{METOdología}

El objetivo de este análisis fue graficar las características de la investigación vinculada al estudio de los periodistas, constatando las particularidades metodológicas y estructurales que han delineado el estado de este tipo de producción científica.

Con base en la búsqueda y análisis de la totalidad de estudios empíricos efectuados en las últimas cinco décadas sobre el periodista latinoamericano, se comparó, a través de una sistematización bibliográfica, el orden formal, espacial y temporal de la diseminación de este tipo de material, los niveles de análisis alcanzados y los enfoques metodológicos seleccionados.

A través del método documental, se estudió la totalidad de trabajos que fueron localizados de forma sistemática, desde 1960 hasta 2007 en 
torno al tema, tras una búsqueda y revisión bibliográfica virtual y presencial en bibliotecas, catálogos y bases de datos validadas dentro del ámbito académico tanto de Latinoamérica como de Estados Unidos y Europa.

Específicamente, se utilizaron las siguientes fuentes de búsqueda y recolección tanto virtual como presencial: Worldcat (catálogo virtual de libros, artículos, libros de actas, reportes de investigación y documentos a nivel mundial), catálogos de las bibliotecas universitarias latinoamericanas, de las bibliotecas universitarias españolas, de bibliotecas universitarias portuguesas, Communication and Mass Media Complete, ComAbstracts, Scientific Electronic Library Online (Scielo); Social Science Citation Index (SSCI); Proquest (tesis y disertaciones); TESEO (Base de datos de tesis doctorales españolas); Latindex, Google Scholar, Catálogo de Documentación en Ciencias de la Comunicación (CC-DOC, en México); PORTCOM (Portal de libre acceso a la producción en ciencia de la comunicación en Brasil); Red de Revistas Científicas de América Latina y El Caribe, España y Portugal (REDALyC); Infoamérica, Dialnet, ISBN, Biblioteca Virtual Cervantes, Centro de Investigaciones Sociológicas (CIS); Banco de Teses Coordenação de Aperfeicoamento de Pessoal de Nivel Superior (CAPES); Tesis electrónicas en línea (Cybertesis) y REDIAL-TESIS.

La revisión de las referencias bibliográficas anexadas en cada uno de los estudios localizados fue otra forma de triangular la búsqueda de los datos, en tanto muchos de los títulos contenidos en dichas bibliografías podrían no haber estado incluidos en los catálogos y bases de datos anteriormente mencionados. De esta forma, se aseguró en mejor medida la representatividad y validez de los textos encontrados, reduciendo el margen de error siempre presente en el rastreo de información en tan amplio espectro espacial y temporal.

Se entendió por investigación sobre la profesión periodística a los estudios empíricos que exploren, describan, correlacionen, comparen o expliquen algún aspecto de la realidad constitutiva del periodista/comunicador y que lo consideren como el objeto de estudio principal del análisis.

Se incluyeron las investigaciones que estudian al periodista desde los diferentes niveles de análisis existentes (individual, organizacional y social), publicadas en los siguientes formatos: revistas científicas, te- 
sis de maestría, tesis de doctorado, libros, capítulos de libros colectivos, reportes de investigación y actas de congresos.

El análisis se circunscribió a las investigaciones escritas tanto en español como en portugués o en inglés, al ser las dos primeras lenguas madres de algún país latinoamericano, y la tercera la segunda más influyente en la región.

Se excluyeron del estudio todos los documentos identificados como textos periodísticos, literarios, de opinión y artículos sobre revisión de libros. Asimismo, a efectos de no restar representatividad al grupo de estudios y publicaciones encontradas, se prefirió focalizar y limitar el cuerpo de análisis, por lo que las tesis de pregrado localizadas fueron finalmente desestimadas dentro del cruce de información, ya que no fue posible tener acceso al cuadro completo de ellas. Sin embargo, las tesis de posgrado sí fueron consideradas en el análisis global, al existir mayores plataformas bibliográficas para su localización, además de ser investigaciones, por lo general, con un nivel suficiente de desarrollo (Vassallo \& Romanchini, 2006, p. 144-145).

Por supuesto, la literatura no publicada, así como la mala distribución de algunos textos que pudieran haber sido incorporados, se transforman en una limitación importante de este estudio. Sin embargo, la triangulación realizada a través de los distintos motores de búsqueda empleados asegura la rigurosidad y exhaustividad de la pesquisa dentro de los soportes validados por este estudio.

Dentro de Latinoamérica se incluyó a todos los países de habla española o portuguesa que, estando geográficamente situados en el norte, centro o sur del Continente, son repúblicas independientes, y que a través de la historia han compartido una herencia colonial, así como estructuras sociales comparables. ${ }^{3}$ A saber: Argentina, Bolivia, Brasil,

3 De acuerdo a Fuentes (1992, p. 62-63), el Continente Americano está dividido geopolíticamente en 45 territorios, cuya administración y gobierno remite a soberanías distintas. La historia colonial ha dejado sus huellas en el hemisferio: 10 de dichos territorios siguen sujetos a regímenes de dependencia directa de otras naciones y los 35 restantes gozan de independencia formal. De estos 35 países independientes, 20 se consideran "latinoamericanos" y los otros 15 de orígenes no-latinos, casi todos anglosajones. 
Chile, Colombia, Costa Rica, Cuba, Ecuador, El Salvador, Guatemala, Honduras, México, Nicaragua, Panamá, Paraguay, Perú, República Dominicana, Uruguay y Venezuela. A pesar de su estrecha vinculación con Estados Unidos, se incluye también a Puerto Rico. ${ }^{4}$

Las unidades de análisis codificadas y utilizadas, a efectos de este primer reporte cuantitativo de la investigación, fueron el estudio y la publicación. Se entendió como estudio al grupo de datos obtenidos por una investigación en particular, mientras que por publicación a los trabajos -léase artículos, libros, tesis, etc.- que emergieron en torno a dichos datos.

Con el objeto de analizar las características de esta evolución se delinearon y operacionalizaron diversos indicadores, tanto a nivel estructural y morfológico de las publicaciones como en contenido y metodología de los estudios. Las categorías de análisis se vincularon a la evolución temporal de las investigaciones, su procedencia, el tipo de formato de publicación, las entidades difusoras más importantes, la tipología temática, los niveles de análisis y los enfoques metodológicos utilizados.

Debido al amplio periodo histórico abarcado por el estudio, el tiempo fue una variable que tuvo especial importancia. Consecuentemente, se utilizaron categorías extensas, dividiendo estos periodos en cinco, coincidentes con cada década analizada. Como veremos a continuación, la cantidad de investigación, aunque en cantidades pequeñas, creció de forma irregular y con variaciones notables durante los 47 años examinados.

\section{RESULTADOS}

\section{Procedencia de los estudios}

El primer paso del análisis fue identificar el número de estudios y publicaciones empíricas efectuadas sobre el periodista latinoamericano, desde los inicios de los estudios de comunicación en la región hasta la

4 Como la definición es "histórica" y por lo mismo "ideológica" - pues trata de identificar la diferencia con respecto a los anglo (y franco) parlantes- la situación deja en la indefinición a Haití, pero incluye a Puerto Rico, aunque no sea un país independiente (entrevista personal con Raúl Fuentes Navarro, académico del ITESO, 2007). 
fecha. En las últimas cinco décadas se encontraron 191 estudios y 231 publicaciones, en tanto 29 investigaciones difundieron sus resultados a través de más de una publicación (véase Gráfica 1).

\section{GRÁFICA 1}

NÚMERO DE PUBLICACIONES POR CADA ESTUDIO

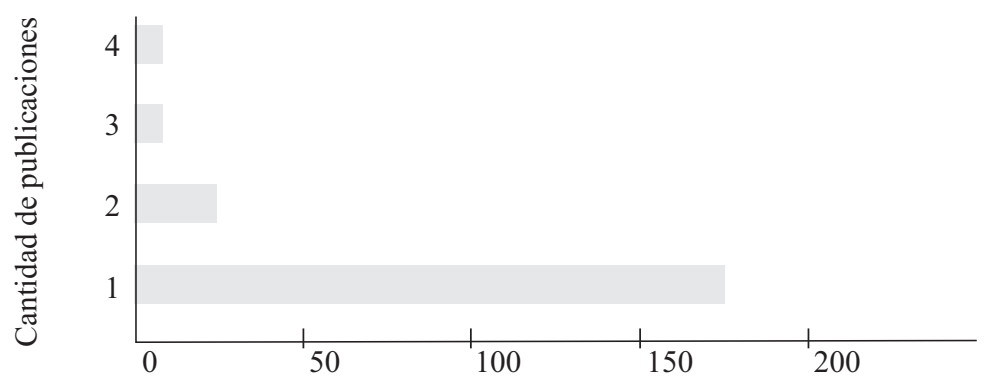

Número de estudios

A causa de la poca cantidad de estudios realizados en las dos primeras décadas, el análisis del total de trabajos enseña que durante el periodo 1980-1989 la producción creció en 750\%, mientras que en los años noventa aumentó en $196 \%$ en comparación con las tres décadas previas. En tanto, durante los últimos siete años se observó un incremento de casi $100 \%$ sobre el periodo anterior, y de $43.1 \%$ en torno al conjunto de las cuatro décadas precedentes.

Pero aunque los años ochenta presentan el mayor crecimiento en términos porcentuales, es desde los noventa, y sobre todo desde el año 2000 en adelante, cuando se comienza a generar la mayor cantidad de trabajos publicados, con un punto máximo en el periodo 2002-2006, donde el año 2003 figura como el más productivo, con 10.4\% del total de los estudios y publicaciones. En efecto, entre la década de 1960 y de 1980, sólo se publicó $10.5 \%$ de toda la producción, mientras que $89.5 \%$-tanto de los estudios como de las publicaciones- se ha generado desde 1990 en adelante, y casi $60 \%$ de ellas ha surgido en los últimos siete años (véase Tabla 1).

En términos de la procedencia de esta productividad, 13 países latinoamericanos y tres extranjeros de habla inglesa, portuguesa o española 
TABLA 1

PRODUCTIVIDAD CIENTÍFICA, PERIODO 1960-2007

\begin{tabular}{cc} 
Décadas & Porcentaje \\
\hline $1960-1969$ & 2.2 \\
$1970-1979$ & 0.9 \\
$1980-1989$ & 7.4 \\
$1990-1999$ & 30.6 \\
$2000-2007$ & 58.9 \\
\hline
\end{tabular}

figuran con publicación de material empírico vinculado al estudio de los periodistas de la región. No obstante, siete países locales no presentan investigación publicada dentro de los soportes consultados desde 1960 hasta 2007, la mayoría de ellos centroamericanos. A saber: Paraguay, República Dominicana, Nicaragua, Panamá, El Salvador, Cuba y Honduras.

De acuerdo a la evolución que las productividades nacionales han tenido a lo largo de las cinco décadas estudiadas -y aunque las cantidades nominales son pequeñas- se observa un importante crecimiento de Chile, país que aumentó en 14 veces su productividad en los últimos siete años en comparación a los noventa. En el mismo período, Brasil casi triplica la cantidad de investigaciones realizadas, liderando este tipo de estudios. En el caso de México -segundo país con mayor número de trabajos publicados- se mantiene estable la productividad en ambas décadas.

Es necesario puntualizar que la supremacía de Brasil se da, en parte, producto de la cantidad de tesis de posgrado efectuadas en dicho territorio. Si se excluye del análisis la publicación de tesis doctorales y de magíster (maestría), Brasil pasa a ocupar el segundo lugar después de México.

Otros países que desde 1990 a la fecha doblan la cantidad de trabajos sobre el tema son España y Estados Unidos. Este último, además, es el único que presenta trabajo investigativo empírico desde la década de 1960. Situación opuesta se observa en el caso de Venezuela, país que en los últimos 17 años ha disminuido su productividad en torno al estudio de los periodistas, en $81.8 \%$.

Independiente de la década de estudio, la tendencia de los investigadores en los países analizados es efectuar investigación empírica en torno a los periodistas, circunscrita casi en su totalidad a su realidad 
nacional y local. En efecto, la gran mayoría de ellos sólo presenta investigación concerniente a sus propios territorios. Los autores extranjeros interesados en Latinoamérica son los que logran diversificar su marco de análisis a distintos espacios geográficos (véase Tabla 2).

\section{TABLA 2}

PRODUCTIVIDAD DE LOS PAÍSES SEGÚN ZONA DE ESTUDIO

\begin{tabular}{lcc} 
& $\begin{array}{c}\text { Publicación referente } \\
\text { al propio país (en \%) }\end{array}$ & $\begin{array}{c}\text { Publicación total } \\
\text { en el país (en \%) }\end{array}$ \\
\hline Estados Unidos & no corresponde & 11.3 \\
Venezuela & 7.8 & 7.8 \\
México & 18.3 & 21.2 \\
Chile & 6.1 & 6.5 \\
Colombia & 2.6 & 3.5 \\
Argentina & 1.7 & 1.7 \\
Perú & 0.9 & 1.7 \\
Holanda & no corresponde & 0.4 \\
Bolivia & 0.4 & 0.4 \\
Guatemala & 0.9 & 0.9 \\
España & no corresponde & 6.1 \\
Brasil & 31.7 & 33.8 \\
Costa Rica & 2.2 & 2.6 \\
Ecuador & 0 & 0.4 \\
Uruguay & 0 & 0.4 \\
Alemania & $72.6 *$ & 0.9 \\
Puerto Rico & no corresponde & 0.4 \\
Total & 0.4 & 100.0 \\
\hline
\end{tabular}

*El porcentaje total es menor a $100 \%$, producto de que no todos los países y zonas geográficas estudiadas corresponden a países en los que se han publicado dichos resultados.

De esta forma, mientras más de $60 \%$ de las investigaciones tienen un alcance local y/o nacional, y $25 \%$ se vincula a estudios de caso, sólo $11.7 \%$ de ellas consiguen un alcance cross nacional. Aún más, es posible observar que desde los noventa desaparecen los estudios cross 
nacionales, para instaurarse mayoritariamente los estudios locales, a pesar de la tendencia comparativa que hoy comienza a posicionarse en el espacio investigativo mundial dentro del periodismo.

\section{Tipo de publicaciones}

Los estudios sobre el periodista latinoamericano fueron clasificados de acuerdo al tipo de publicación, dividiéndose en siete formatos distintos, según indica la Tabla 3.

\section{TABLA 3}

TIPOS DE PUBLICACIÓN

\section{Porcentaje}

\begin{tabular}{lc}
\hline Artículos académicos & 35.1 \\
Libros & 11.3 \\
Libros de actas & 3.0 \\
Tesis doctorales & 11.3 \\
Tesis de magíster & 22.9 \\
Capítulos de libros & 9.5 \\
Reportes de investigación & 6.5 \\
Otros & 0.4 \\
Total & 100.0 \\
\hline
\end{tabular}

De estos datos se desprende el predominio del artículo como forma de difusión del conocimiento en torno al periodista, seguido muy de cerca por las tesis de posgrado. En efecto, los artículos publicados se incrementaron en más del doble en la última década con respecto a la anterior, publicándose 17 veces más si lo comparamos con los años ochenta. Una situación similar es la ocurrida con las tesis de magíster y de doctorado, las cuales crecieron durante los últimos siete años, en $94 \%$ y $260 \%$ respectivamente, en comparación con los 10 años inmediatamente anteriores.

En contraposición, los libros sufrieron una baja importante en la última década, en la cual se publicó la mitad de títulos que se editaron en los noventa. Los capítulos de libro, no obstante, han mostrado un crecimiento sostenido. 
La supremacía de uno u otro formato sufre ciertos matices de acuerdo al país de publicación. Mientras la distribución en torno a los artículos en revistas y libros de actas es similar a la observada en la productividad global, Venezuela, México y Brasil igualan la cantidad de libros publicados, y México lidera la presencia de capítulos de libros, con más de la mitad de la producción (véase Gráfica 2).

A nivel de tesis doctorales figuran Brasil, Estados Unidos y España, con 12, siete y seis trabajos respectivamente; en tanto Brasil presenta más de $60 \%$ de las tesis de magíster efectuadas en torno al estudio de los periodistas. Finalmente, Chile figura con la mayor cantidad de reportes de investigación, seguido por Colombia y Perú.

\section{GRÁFICA 2}

TIPOS DE PUBLICACION EN PAÍSES CON MAYOR PRODUCTIVIDAD

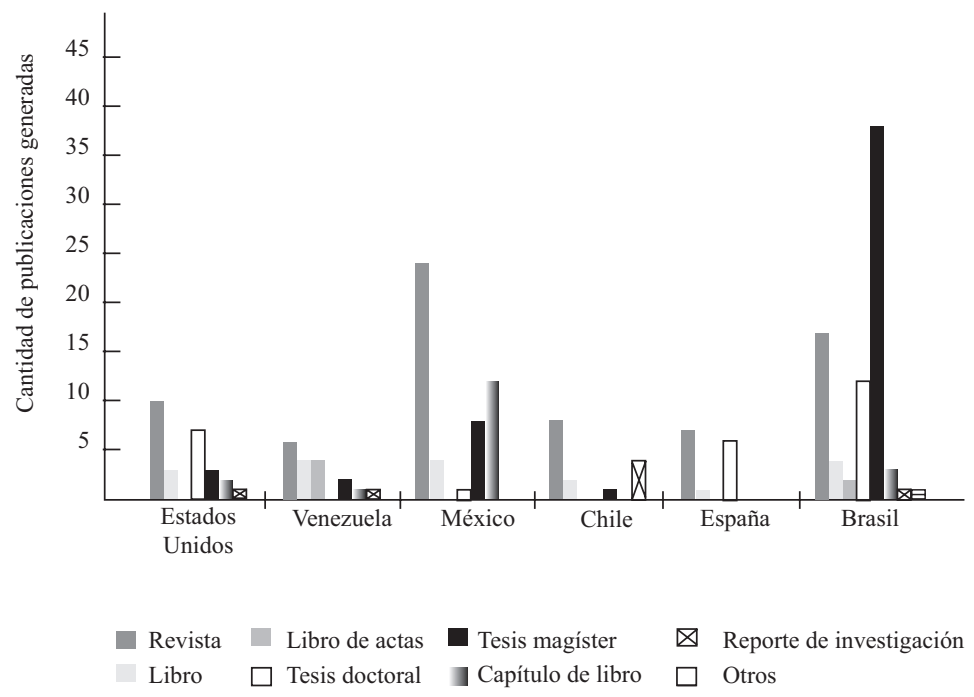

\section{Difusión de los estudios}

Se localizaron 135 entidades difusoras entre las publicaciones analizadas. Estas corresponden a 46 universidades, 45 revistas, 21 editoriales y 23 instituciones y/o asociaciones vinculadas al periodismo. Mientras 
que las primeras han aportado con $43.3 \%$ de la publicación de trabajos en el área, las revistas lo han hecho con $32 \%$; las editoriales, con $11.3 \%$, y las asociaciones con $13 \%$ de la producción.

Dentro de las entidades de educación superior, la Universidad de São Paulo lidera con 24 publicaciones (en su mayoría tesis de magíster y doctorado), seguida con distancia por la Universidad de Guadalajara de México con cinco trabajos; la Universidad Federal de Río de Janeiro con cuatro, y las universidades de Brasilia, Internacional de Florida (Estados Unidos), Iberoamericana (México) y La Laguna (España), con tres publicaciones cada una. En el contexto de las editoriales, Nueva Imagen figura con tres publicaciones, y a nivel de instituciones, la Fundación Konrad Adenauer con cuatro.

De acuerdo a los estudios de referencia que muchos investigadores utilizan en sus análisis bibliográficos, aproximadamente la mitad de los artículos sobre cualquier tópico están concentrados en una docena y tanto de revistas; mientras que el resto está disperso extensamente en cientos de revistas (Swanson, 1966). Dicha situación -contrastada en contextos diversos- es aún más restringida en este caso, donde apenas seis revistas logran figurar dentro del total a nivel individual, aunque con un bajo número de publicaciones vinculadas y con una falta de continuidad temporal a través de las décadas en estudio.

En el caso de la Revista de Comunicación Estudios Venezolanos, su productividad en torno al tema se concentra en exclusiva en la década de 1990; mientras que en Sala de Prensa, UNI Revista, Razón y Palabra y Em Questão, se extiende desde los noventa hasta la fecha. Journalism Quarterly (hoy Journalism \& Mass Communication Quarterly), en tanto, se posicionó como mayor difusor de estos estudios sólo en los años sesenta. En efecto, los cuatro artículos emergidos durante esa década se publicaron allí (véase Tabla 4).

Asimismo, sólo $11.1 \%$ de los artículos generados durante las cinco décadas en estudio fueron publicados en revistas de corriente principal, indexadas al Social Science Citation Index (SSCI). Una de las razones que podrían explicar dicha situación es el manejo de la segunda lengua -ya que la mayoría de estas revistas son publicadas en inglés-, así como la falta de competencias para enfrentar los altos estándares metodológicos y conceptuales exigidos por dichas publicaciones. 


\section{TABLA 4}

REVISTAS CON MAYOR NÚMERO DE PUBLICACIONES EMPÍRICAS SOBRE EL PERIODISTA LATINOAMERICANO

Revistas Número de artículos publicados

\begin{tabular}{ll}
\hline Revista Comunicación & \\
Estudios Venezolanos & 6 \\
Journalism Quarterly & 6 \\
Sala de Prensa & 6 \\
UNI Revista & 4 \\
Razón y Palabra & 5 \\
Em Questão & 3 \\
\hline
\end{tabular}

Llama la atención que las revistas más prestigiosas dentro de los estudios de comunicación en Latinoamérica, como Diálogos de la Comunicación, Signo y Pensamiento, y Comunicación y Sociedad prácticamente no hayan publicado resultados de estudios empíricos en torno al periodista regional. Considerando las tres publicaciones, sólo se encontraron cuatro artículos directamente relacionados.

\section{Temáticas de estudio}

La inexistencia de características previas o sistematizaciones que estipularan de forma concreta qué tipos de estudio y tendencias temáticas se habían desarrollado en torno a la investigación de los periodistas en la región latinoamericana, fue uno de los aspectos que motivó su búsqueda.

Después de un análisis del contenido de cada investigación y de la literatura que permitió profundizar en las estructuras de estos trabajos, se detectaron "diez tipos de estudios” diferenciados (véase Gráfica 3).

1) Los estudios sobre "rutinas y prácticas periodísticas" llevados a cabo a nivel latinoamericano (31.6\% de los casos) se enfocan en cómo los periodistas hacen su trabajo, en las actividades diarias que desarrollan y en la elaboración de los productos informativos (herramientas más usadas, rutinas de búsqueda, contactos y manejo de información), así como en los problemas y circunstancias particulares que viven en el ejercicio de su profesión. 
2) Los estudios sobre el "perfil ocupacional y las condiciones del mercado laboral" (19.3\% de los casos) suelen orientarse -aunque no en exclusiva- al análisis de periodistas recién egresados, explorando la oferta y demanda de estos profesionales, el número de titulados en las escuelas de comunicación, la colocación y la empleabilidad real de los periodistas, así como las condiciones de trabajo de los recién titulados. En este sentido, algunos de ellos logran operacionalizar los distintos nichos laborales donde el periodista se desempeña o podría desempeñarse, así como los criterios que utiliza el empleador para estimar los roles y funciones que pueden efectuar estos profesionales.

3) Los estudios sobre la "formación del periodista" (18.7\% de los casos) se orientan en su mayoría a la evaluación que los propios sujetos hacen de la enseñanza recibida en las universidades, las relaciones existentes entre la formación y la práctica profesional, así como las competencias requeridas para el desarrollo de su labor.

4) Los estudios sobre "roles profesionales" (16\% de los casos) pretenden conocer cuáles son los principios, valores, influencias y actitudes que guían a los periodistas en su comportamiento diario, asociado con la información que generan. En este sentido, este tipo de investigación se vincula a la detección de características, percepciones y posturas éticas del periodista frente a situaciones puntuales y presiones vinculadas a su trabajo, así como a la función del periodismo en la sociedad. Desde este enfoque, es importante la forma en que los periodistas se ven a sí mismos y las decisiones que toman frente a determinados temas.

5) Los estudios sobre las "condiciones laborales del periodista" $(15.5 \%$ de los casos) se vinculan a la satisfacción del profesional con su entorno laboral general. Sin embargo, las investigaciones encontradas están más orientadas a analizar las condiciones físicas y materiales de su trabajo (horarios, salarios, etc.), que los aspectos vinculados al ambiente laboral, al estrés y al bienestar emocional.

6) Los estudios sobre las "representaciones sociales del periodista" $(10.7 \%$ de los casos) suelen ser investigaciones aplicadas a estudiantes de periodismo o a empleadores de periodistas, quienes describen y conceptualizan la formación, funciones y roles del sujeto 
profesional, de acuerdo al imaginario por ellos generado. Asimismo, estos estudios se orientan a conocer la autorrepresentación y estructuración de la identidad profesional de los periodistas. Muchos de ellos, además, exploran las formas que emplean los profesionales y estudiantes para justificar sus opciones profesionales, de acuerdo a la representación social que tengan del periodismo.

7) Los estudios sobre el "perfil sociodemográfico del periodista" (8.6\% de los casos) se limitan a describir las características personales que definen al sujeto como ser humano y trabajador. Por lo general tienen un propósito mayor, que es vincular dichos factores a las condiciones laborales, a las actitudes profesionales o al mercado laboral del periodista.

8) Los estudios encontrados sobre las "condiciones asociativas del periodista" están presentes en $5.3 \%$ de los casos y se orientan a conocer las impresiones que aquellos poseen respecto a sus derechos como trabajadores, al rol que las asociaciones y colegios deben tener en torno al buen ejercicio de la profesión, así como a encuestas vinculadas a cifras de colegiación.

9) El estudio sobre la "orientación profesional del periodista" $3.7 \%$ de los casos) está circunscrito a conocer, desde el enfoque de la sociología de las profesiones y a través de escalas y medidas establecidas, los niveles de profesionalización y de desarrollo alcanzados por estos profesionales y por el periodismo en general.

10) Finalmente, el "estudio de género dentro de la profesión periodística" (3.2\% de los casos) hace mención explícita de las diferencias que hombres y mujeres tienen en el ejercicio de la profesión.

De acuerdo a los datos encontrados, el tipo de investigación más desarrollada se orienta al estudio de las rutinas y prácticas periodísticas. Le siguen la investigación sobre el perfil ocupacional y las condiciones del mercado laboral, y los estudios sobre la formación del periodista. En el otro extremo, las tipologías menos desarrolladas han sido el estudio sobre la orientación profesional y los niveles de profesionalización de los periodistas, así como el estudio de género dentro de la profesión.

La evolución de la investigación, a través de los años, muestra que los estudios efectuados en torno a las rutinas y prácticas profesionales 
han sido una de las líneas de trabajo de más grande desarrollo desde los años noventa, cuyo número se ha doblado en los últimos siete años. Una situación similar sucede con los estudios sobre representaciones e imaginarios sociales del periodista, los cuales han crecido desde seis -encontrados en la década de 1990-a 13 investigaciones vinculadas en los últimos siete años.

El análisis efectuado permite, además, analizar el predominio de determinados tipos de estudios en los distintos países que estudian la región, década tras década. En este sentido, nos encontramos con que si bien durante los años sesenta y setenta Estados Unidos fue superior en todos los tipos de estudios efectuados, durante los ochenta Venezuela lideró la investigación sobre las condiciones laborales del periodista, mientras que Estados Unidos encabezó los estudios sobre formación. El panorama cambió radicalmente desde los noventa en adelante, donde Brasil y México comparten protagonismo en el desarrollo de todas las tipologías más desarrolladas.

\section{Niveles de análisis}

En términos generales, se puede hablar de tres niveles de análisis dentro del estudio del periodismo, teóricamente definidos, pero no tan fácilmente diferenciados en la práctica. En el primero, el interés se centra en las creencias, valores, experiencias y conocimientos que los periodistas poseen y aplican en una determinada actividad laboral. Asimismo, importa conocer y contextualizar su formación y características como profesional, sus roles y actitudes profesionales, su realidad laboral, sus actitudes frente a la asociatividad y las características personales (sexo, edad, constitución familiar, nivel socioeconómico, etc.) que los definen. En el segundo, el interés se concentra en las características de los lugares de trabajo-estructuras, rutinas y condiciones características- así como en el análisis de las organizaciones y entidades formativas que "profesionalizan" al periodista. La tercera perspectiva apunta hacia el conocimiento de las estructuras sociales que interactúan de forma sistémica -aunque no siempre de forma simétrica-con el periodismo a nivel mundial.

En la literatura teórica y metodológica vinculada a esta temática, encontramos diferentes posturas y discusiones respecto a la importancia de análisis que cada nivel comprende por separado. Están quienes 
GRÁFICA 3

TIPOS DE ESTUDIOS EN PAÍSES CON MAYOR PRODUCTIVIDAD

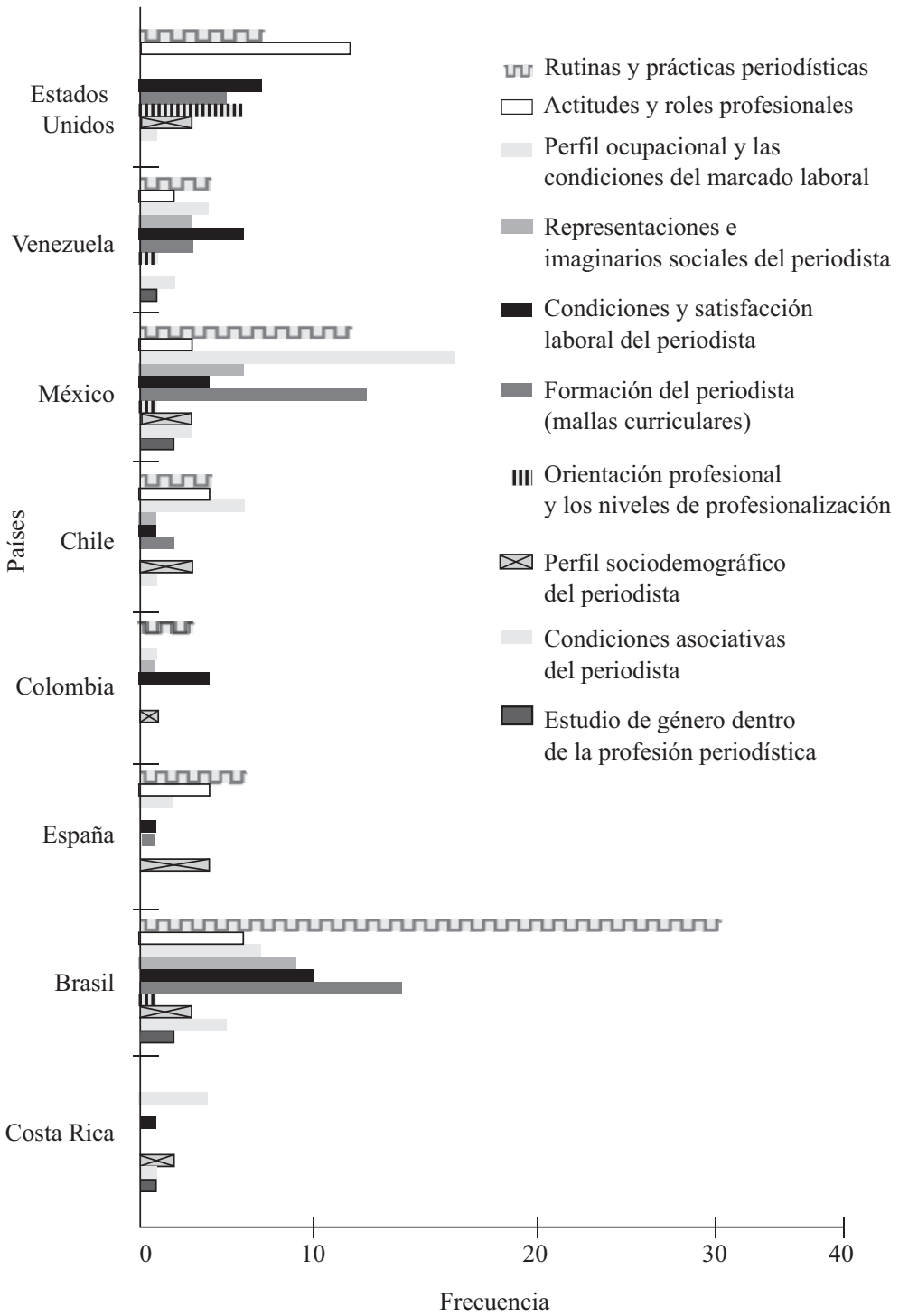


apelan a la necesidad de aumentar los estudios efectuados desde una óptica organizacional, en tanto como nivel de análisis podrían explicar una mayor cantidad de la varianza en torno al comportamiento, producción y mediación social del periodista. Por otro lado, están quienes defienden el nivel individual desde una perspectiva psicológica, indicando que la influencia de factores personales en la producción de contenido es un aspecto importante y, en algunos casos, decisivo (Donsbach, 2008; Kepplinger \& Rouwen, 2000). Finalmente, aparecen quienes, desde la teoría de sistemas sociales, defienden como fundamental los marcos y estructuras sociales que sostienen al periodismo en diferentes regiones del globo (Ruhl, 2008).

Del análisis aquí efectuado es posible observar que más de $60 \%$ de los estudios empíricos sobre el periodista latinoamericano ponen su atención en los factores internos que delimitan y condicionan al sujeto más que en los agentes intermedios o en los factores externos que contextualizan su ejercicio profesional. Aún más, dentro de aquellos factores existe un claro desequilibrio a favor de la realidad laboral y de la formación del periodista, en contra del desarrollo de sus prácticas asociativas y de sus niveles de profesionalización. Les sigue $31.8 \%$ de los estudios que logran vincular tanto la actuación de los factores internos como intermedios, aunque con un bajo porcentaje de estudios que incorporen el ámbito de las asociaciones y gremios de periodistas.

Sólo tres estudios (1.7\%) articulan, desde una visión panorámica global, tanto los factores internos, intermedios como externos del modelo. Es decir, la interrelación del estudio de las características de los "periodistas" que se desempeñan como tales; del estudio de las "organizaciones" en el marco de las cuales los periodistas desarrollan su actividad (empleadores, asociaciones y facultades de comunicación) y del marco social, político y económico que delimita el contexto latinoamericano.

\section{Enfoques metodológicos}

La preponderancia de la investigación cuantitativa y/o cualitativa dentro de los estudios de periodismo ha incentivado a muchos estudiosos alrededor del mundo a efectuar mediciones y buscar patrones en la productividad generada. 
En este estudio, una mayoría de las investigaciones efectuadas sobre el periodista durante las últimas cinco décadas corresponde a enfoques cuantitativos (42.3\%). Sin embargo, dicha mayoría es seguida de cerca por $36.2 \%$ que se aglomera en torno al enfoque cualitativo, y por $21.5 \%$ de casos que utiliza ambos enfoques metodológicos.

Dichos porcentajes presentan importantes variaciones, sobre todo entre la década de 1990 y la actual, donde los estudios cualitativos aparecieron y se triplicaron en sólo 17 años; los estudios mixtos se duplicaron, mientras que los estudios cuantitativos tuvieron una baja marginal. Las ponderaciones encontradas no dejan de sorprender en tanto los estudios cualitativos se posicionan como el enfoque más utilizado en los últimos siete años, superando al enfoque cuantitativo en $18.5 \%$, incluso cuando, previo a la década de 1990 no se habían presentado investigaciones que utilizaran sólo ese enfoque (véase Tabla 5).

Estos resultados coinciden con lo encontrado por Fuentes (1996, 2004) en torno a la producción en comunicación en México, aunque contradicen hallazgos de estudios que han analizado el tipo de material publicado en revistas de gran influencia a nivel internacional (Cooper, Dupagne\& Potter, 1994; Kamhawi \& Weaver, 2003).

\section{TABLA 5}

INVESTIGACIÓN CUANTITATIVA Y CUALITATIVA

EN LA PRODUCCIÓN CIENTÍFICA 1990-2007

Cualitativo Cuantitativo Cuantitativo/ Total

Cualitativo

\begin{tabular}{rrrrr}
\hline $1990-1999$ & $18(27.8 \%)$ & $35(55.6 \%)$ & $11(16.7 \%)$ & $64(100 \%)$ \\
$2000-2007$ & $51(47.8 \%)$ & $31(29.3 \%)$ & $24(22.8 \%)$ & $106(100 \%)$ \\
\hline
\end{tabular}

De dicha situación pueden desprenderse varias interpretaciones. Por un lado, se puede enfrentar la generalización del interés de los estudios cualitativos en la comunidad científica latinoamericana, situación que coincide con el predominio y aumento de los estudios sobre rutinas y prácticas periodísticas. Sin embargo, también puede delatar una falta de conocimiento de los nuevos investigadores sobre la aplicación y el uso de técnicas cuantitativas en la investigación científica. 
En esta línea, ciertos países tienden a presentar mayor investigación cualitativa. Es el caso de Brasil, donde sólo $6.73 \%$ de su productividad en el tema utiliza un enfoque cuantitativo, y $20 \%$ utiliza un enfoque mixto.

En Venezuela (75\%), Chile (66.7\%) y Argentina (80\%), predomina la investigación cuantitativa en torno a este tipo de estudios.

\section{DISCUSIÓN}

A lo largo de esta sistematización bibliográfica documental se describieron las principales características estructurales y metodológicas que han definido el estado de la investigación sobre el periodista latinoamericano en las últimas cinco décadas.

Los resultados enseñan, en primera instancia, un incremento notable en la productividad científica generada en torno a este tipo de investigaciones. No obstante, aún no se puede hablar de una consolidación de este ámbito de estudios, ni a nivel latinoamericano, ni en los referentes nacionales y locales que los componen. De acuerdo a los datos analizados, se observa un crecimiento tardío en el área. Tanto es así que $60 \%$ del material se ha generado en los últimos siete años.

Las productividades por país tampoco han sido homogéneas. Coincidiendo con una exploración cualitativa previa de este material (Mellado, 2009), los datos aquí analizados muestran que mientras Brasil y México -y en menor medida Venezuela y Chile- destacan por su productividad, la mayoría de los países centroamericanos permanecen ausentes en este tipo de estudios.

Los problemas que han atravesado el desarrollo del campo de la investigación en comunicación en la región, tales como falta de recursos humanos y de investigación, el desconocimiento de la producción latinoamericana y la hibridez conceptual y metodológica, podrían explicar la aún incipiente investigación empírica diseminada a través de los formatos encontrados. Este aspecto es preocupante, en tanto muchos de los países mencionados no estarían efectuando esfuerzos suficientes por conocer de forma sistemática a sus periodistas como grupo ocupacional y profesional. La situación se vuelve más compleja a la hora de querer homologar criterios para efectuar estudios comparativos con otras regiones. 
Todo aquello demuestra el retraso y la irregularidad que Latinoamérica ha tenido en su productividad científica en torno al periodista, así como el impacto que dicha demora ha significado en el desarrollo de los estudios de periodismo.

En términos de la diseminación del material analizado figura la supremacía del artículo y la disminución del libro como forma general de publicación, situación que se explica por la falta de recursos y de editoriales disponibles para tales efectos, así como por la aparición de diversas revistas y publicaciones locales.

Paralelamente, los resultados enseñan la transición desde un enfoque metodológico marcadamente cuantitativo utilizado en la investigación científica-empírica sobre el periodista, a un carácter mayoritariamente cualitativo de este tipo de estudios en la última década.

Uno de los hallazgos importantes de este trabajo se vincula con los niveles de análisis utilizados en el estudio del periodista. De acuerdo a los datos, la investigación no logra hacerse cargo de todos los factores por igual, priorizando el estudio individual del periodista por sobre los modelos multinivel de análisis. Así es como en términos macrosociales casi no se han hecho esfuerzos por analizar al periodista, por lo que la investigación y la discusión teórica en torno a él aún se encuentra en un micronivel de análisis, donde la interrelación de los agentes externos, intermedios e internos que condicionan y delimitan su concepción aún no ha podido ser desarrollada.

Esto provoca, sin duda, una descontextualización de los elementos constituyentes del ejercicio cotidiano y de las vivencias más próximas del sujeto profesional, al no considerarse los condicionantes del sistema en su totalidad.

Finalmente, la mayoría de los estudios efectuados sobre el periodista regional no logran involucrar el esfuerzo colaborativo de más de un país. De acuerdo a Hantrais \& Mangen (1996), pocos científicos sociales se sienten bien preparados para conducir estudios cross nacionales o trabajar en equipos internacionales; renuencia que puede ser explicada no sólo por una carencia de conocimiento o entendimiento de culturas y lenguas diferentes, sino también por la insuficiente conciencia de las tradiciones del proceso de la investigación que se desarrolla en espacios nacionales distintos. 
En este contexto, cobran absoluta validez las críticas de Beltrán (1977), quien ha resaltado la falta de un mínimo grado de sistematización que facilite el uso de los resultados en más de un país, generándose una carencia de coordinación que duplica esfuerzos y desaprovecha experiencias locales.

América Latina está aún lejos de alcanzar los niveles de ciencia y tecnología que caracterizan a la mayoría de los países desarrollados en el ámbito de los estudios de la comunicación y el periodismo. En efecto, la especialidad de investigación sobre la profesión periodística aún permanece difusa y desestructurada.

Por esta razón, la región requiere fomentar con urgencia la actividad científica para impulsar su desarrollo económico, político y social, y para comenzar a desarrollar ámbitos académicos como este.

Considerando los grandes cambios tecnológicos, económicos y sociales vinculados a la profesión periodística, sería improbable pensar que no sigan apareciendo nuevos conceptos y teorías que la remodelen y redefinan. Lo importante, sin embargo, es que esto suceda a la par de una correlativa madurez y reconocimiento de la historia y de las estructuras que sostienen su evolución dentro del continente latinoamericano.

\section{Bibliografía}

Beltrán, L. R. (1977). La investigación en comunicación en América Latina. ¿Indagación con anteojeras? Órbita, 21, 5-56.

Cooper, R., Dupange, M. \& Potter, J. (1994). A Status Report on Methods Used in Mass Communication Research. Journalism Educator, 48, 54-61.

Donsbach, W. (2008). Factors behind Journalists' Professional Behavior. A psychological Approach to Journalism Research. En M. Lôffelholz \& D. H. Weaver (Eds.), Global Journalism Research. Theories, Methods, Findings, Future (pp. 65-78). Malden, MA: Blackwell Pub.

Fuentes, R. (1991). La comunidad desapercibida. Investigación e investigadores de la comunicación en México. México: ITESO.

Fuentes, R. (1992). Un campo cargado de futuro. El estudio de la comunicación en América Latina. México: FELAFACS.

Fuentes, R. (1996). La investigación de la comunicación en México. 
Sistematización documental 1986-1994. México: Universidad de Guadalajara e ITESO.

Fuentes, R. (Coord.) (2004). Producción, circulación y reproducción académicas en el campo de la comunicación en México. México: ITESO.

Hantrais, L., \& Mangen, S. (Eds.) (1996). Cross-national research methods in the social sciences. London: Pinter.

Kamhawi, R., \& Weaver, D. (2003). Mass Communication Research Trends from 1980 to 1999. Journalism \& Mass Communication Quarterly, 80 (1), 7-27.

Kepplinger, H. M., \& Rouwen, B. (2000). Der prognostische Gehalt der Nachrichtenwert-Theorie (The prognostic power of the theory of newsworthiness), Publizistik, 45, 462-475.

Meditsch, E., \& Segala, M. (2005). Trends in three 2003/4 journalism academic meetings. Brazilian Journalism Research, 1 (1), 47-60.

Mellado, C. (2009). Orígenes, evolución y desencuentros en la investigación sobre el periodista latinoamericano. Innovar, 19 (33), 7-17.

Menanteau-Horta, D. (1967). Professionalism of Journalists in Santiago de Chile. Journalism Quarterly, 44, 15-23.

Ruhl, M. (2008). Journalism in a Globalizing World Society. A Societal Approach to Journalism Research. En M. Lôffelholz \& D. H. Weaver (Eds.), Global Journalism Research. Theories, Methods, Findings, Future (pp. 65-78). Malden, MA: Blackwell Pub.

Swanson, D. R. (1966). Scientific Journals and Information Services of the Future. American Psychologist, 21, 1005-1010.

Tuchman, G. (1978). Making News. A Study in the Construction of Reality. New York: Free Press.

Vassallo, M. I., \& Romanchini, R. (2006). Teses e Dissertações: Estudo Bibliométrico na Área da Comunicação. En D. Aguiar de Población, G. Porto Witter, \& J. F. Modesto de Silva (Coords.), Comunicação e produção científica: contexto, indicadores y avalidação (Comunicación y producción científica: contexto, indicadores y evaluación) (pp. 137-161). São Paulo: Angellara Editora e Livraria.

Wright, Ch. (1975). Mass Communication: A Sociological Perspective.

New York: Random House. 
